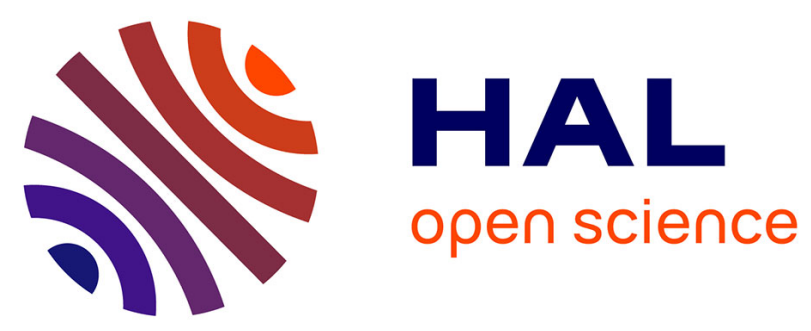

\title{
Numerical homogenization of electrokinetic equations in porous media using lattice-Boltzmann simulations
}

\author{
Amaël Obliger, Magali Duvail, Marie Jardat, Daniel Coelho, Samir Bekri, \\ Benjamin Rotenberg
}

\section{- To cite this version:}

Amaël Obliger, Magali Duvail, Marie Jardat, Daniel Coelho, Samir Bekri, et al.. Numerical homogenization of electrokinetic equations in porous media using lattice-Boltzmann simulations. Physical Review E: Statistical, Nonlinear, and Soft Matter Physics, 2013, 88, pp.013019. 10.1103/PhysRevE.88.013019 . hal-01078978

\section{HAL Id: hal-01078978 \\ https://hal.sorbonne-universite.fr/hal-01078978}

Submitted on 30 Oct 2014

HAL is a multi-disciplinary open access archive for the deposit and dissemination of scientific research documents, whether they are published or not. The documents may come from teaching and research institutions in France or abroad, or from public or private research centers.
L'archive ouverte pluridisciplinaire HAL, est destinée au dépôt et à la diffusion de documents scientifiques de niveau recherche, publiés ou non, émanant des établissements d'enseignement et de recherche français ou étrangers, des laboratoires publics ou privés. 


\title{
Numerical homogenization of electrokinetic equations in porous media using lattice-Boltzmann simulations
}

\author{
Amaël Obliger, ${ }^{1,2, *}$ Magali Duvail, ${ }^{3}$ Marie Jardat, ${ }^{1}$ Daniel Coelho, ${ }^{2}$ Samir Békri, ${ }^{4}$ and Benjamin Rotenberg ${ }^{1}$ \\ ${ }^{1}$ CNRS, UPMC Univ. Paris 06, UMR 7195 PECSA, 75005 Paris, France \\ ${ }^{2}$ Andra, Parc de la Croix-Blanche, 1-7, rue Jean-Monnet, 92298 Châtenay-Malabry cedex, France \\ ${ }^{3}$ Institut de Chimie Séparative de Marcoule, UMR 5257, CEA CNRS UM2 ENSCM, F-30206 Bagnols Sur Cèze, France \\ ${ }^{4}$ IFP-Energies nouvelles, 1-4 Avenue de Bois-Préau, Rueil-Malmaison 92852, France
}

(Received 27 February 2013; revised manuscript received 14 May 2013; published 31 July 2013)

\begin{abstract}
We report the calculation of all the transfer coefficients which couple the solvent and ionic fluxes through a charged pore under the effect of pressure, electrostatic potential, and concentration gradients. We use a combination of analytical calculations at the Poisson-Nernst-Planck and Navier-Stokes levels of description and mesoscopic lattice simulations based on kinetic theory. In the absence of added salt, i.e., when the only ions present in the fluid are the counterions compensating the charge of the surface, exact analytical expressions for the fluxes in cylindrical pores allow us to validate a new lattice-Boltzmann electrokinetics (LBE) scheme which accounts for the osmotic contribution to the transport of all species. The influence of simulation parameters on the numerical accuracy is thoroughly investigated. In the presence of an added salt, we assess the range of validity of approximate expressions of the fluxes computed from the linearized Poisson-Boltzmann equation by a systematic comparison with LBE simulations.
\end{abstract}

DOI: 10.1103/PhysRevE.88.013019

PACS number(s): 47.57.-s, 47.56.+r, 47.60.-i, 47.61.-k

\section{INTRODUCTION}

Electrokinetic effects refer to the dynamic coupling between the solvent and charge flows which occur at a charged interface. Such solid-liquid or liquid-liquid interfaces are ubiquitous and electrokinetic effects are known to play an important role in many contexts, such as colloidal science [1], microfluidics [2-4], biology [5-7] or, on a larger scale, geophysics [8,9]. As an example, electro-osmosis generates a solvent flow under an applied electric field, due to the driving of the electrically charged fluid in the vicinity of charged surfaces. Conversely, a pressure gradient induces the flow of a charged fluid, hence, an electric current. The presence of surface charge in a porous medium has important practical applications in membrane technology (e.g., ion exchange and water desalination) and in environmental science, since most rocks and soils contain minerals (such as clays) that bear a permanent surface charge.

The modeling and simulation of electrokinetic effects in porous media, and, more generally, of all coupled transport phenomena, including the osmotic solvent flow due to a salt concentration gradient, thus have been the subject of a large number of investigations, both on the pore scale where the couplings originate and on the sample scale corresponding to the experimental measurements. Recently, significant progress has been made on the derivation of the macroscopic transport equations from the pore-scale ones [10-12]. These studies usually start from a continuous description of the fluid via transport equations [Poisson-Nernst-Planck (PNP) and NavierStokes (NS)], which are then upscaled to derive their average effect on the sample scale, which is quantified by a coupling matrix relating the solvent and ionic fluxes to the corresponding forces (pressure, potential, and concentration gradients).

*amael.obliger@gmail.com
From the mathematical point of view, this upscaling can be performed rigorously using the homogenization approach. For instance, Moyne and Murad provided a complete solution of the coupled hydro-electro-chemo-mechanical couplings in clays, within the PNP approximation [13,14]. This provides expressions of the macroscopic transport coefficients as solutions of coupled partial differential equations on the pore scale, which then have to be solved using simplifying assumptions or numerically. Furthermore, they have shown that the coupling matrix, when correctly stated in terms of the appropriate quantities, is symmetric - in line with Onsager's reciprocity relations. Allaire et al. arrived at the same conclusion and further analyzed the convergence of the homogenization procedure [15]. The Onsager symmetry relation had already been demonstrated for the response to pressure and electric potential gradients in the case of networks of cylindrical capillaries by Mazur and Overbeek [16] and later extended to arbitrary pore geometries in the limit of thin double-layers by Brunet and Ajdari [17].

A complementary route to the macroscopic transport coefficient in complex porous media is the direct numerical resolution of the coupled PNP and NS equations for large systems. Adler and coworkers have performed extensive simulations of electrokinetic flows in various porous media, including random packings and reconstructed and fractured porous media $[18,19]$. This approach also allowed us to investigate new regimes such as high $\zeta$ potentials [20] and to evidence a universal behavior if appropriate rescaled quantities are introduced [20,21].

More recently, alternative methods have been proposed to simulate electrokinetic effects starting from a more fundamental description of the fluid than the PNP and NS equation [22]. Molecular simulations have also been used to investigate the limitations of continuous descriptions of electrokinetic effects, especially under extreme confinement [23-29]. However, their computational cost prevents their 
use to simulate large-scale samples. For the simulation of hydrodynamic flows, coarse-grained simulations based on kinetic theory such as the lattice-Boltzmann method [30] have become a standard tool, in particular due to their ability to deal with complex geometries or complex fluids [31-36]. This scheme has also been extended to capture the coupling of hydrodynamic flow with ion transport and the simulation of electrokinetic effects in colloidal suspensions or porous media or at liquid-liquid interfaces [37-41]. Finally, an alternative approach to both extensive numerical simulations and the homogenization strategy is to resort to coarser models of the transport in porous media such as the pore network model [42-44]. In this framework, which has to date never been extended to account for the transport of charged species and of electrokinetic effects, transfer coefficients at the pore scale are the input of the numerical solver and analytical expressions for these quantities are needed.

In this context, the objective of this paper is to derive simple analytic expressions of the transfer coefficients which couple the solvent and ionic fluxes under the effect of pressure, electrostatic potential, and concentration gradients at the pore scale and to assess their range of validity. To achieve this goal, we introduce a variant of the lattice-Boltzmann electrokinetics scheme which also captures the coupled solvent and ionic flows under salt concentration gradients. In a first step, we, first, validate this new scheme against the exact analytical solution of the PNP and NS equation in the absence of added salt, i.e., when the only ions present in the fluid are the counterions compensating the charge of the surface. These simulations then provide a reference to assess the validity of approximate analytic expressions obtained within a linearized Poisson-Boltzmann approach.

The paper is organized as follows. In Sec. II we, first, recall the description of coupled transport phenomena in porous media on the macroscopic (sample) and microscopic (pore) scales. In Sec. III we derive analytical expressions for all transport coefficients in the cylindrical geometry. The latticeBoltzmann electrokinetics (LBE) algorithm is presented in Sec. IV, and the modification allowing for the description of osmosis is introduced. The choice of simulation parameters depending on the system (nanochannel radius and surface charge, salt concentration) is discussed in Sec. V. Finally, all transport coefficients, determined numerically as a function of salt concentration, are presented in Sec. VI.

\section{TRANSPORT ON THE MICRO- AND MACROSCALES}

\section{A. Sample scale}

The transport of an electrolyte solution through a charged porous medium, under the effect of macroscopic pressure $\bar{P}$, electric potential $\bar{\psi}$, or salt concentration $\bar{\rho}$ gradients is usually described in terms of a response matrix relating the macroscopic fluxes of solvent $\bar{J}_{s}^{m}$ (the superscript indicates a mass flux), cations $\bar{J}_{+}$, and anions $\bar{J}_{-}$to the applied forces,

$$
\left(\begin{array}{c}
\bar{J}_{s}^{m} \\
\bar{J}_{+} \\
\bar{J}_{-}
\end{array}\right)=-\left(\begin{array}{ccc}
K_{s}^{P} & K_{s}^{\psi} & K_{s}^{\rho} \\
K_{+}^{P} & K_{+}^{\psi} & K_{+}^{\rho} \\
K_{-}^{P} & K_{-}^{\psi} & K_{-}^{\rho}
\end{array}\right)\left(\begin{array}{c}
\nabla \bar{P} \\
\nabla \bar{\psi} \\
k_{B} T \nabla \ln \bar{\rho}
\end{array}\right)
$$

In principle, each element is itself a tensor [13,14]. In the following, we will restrict our discussion to the case of a cylindrical channel, for which only axial flows are observed and, hence, the coupling elements defined above are scalar quantities. The coupling matrix includes both diagonal terms, such as the permeability $K_{s}^{P}$, and nondiagonal ones reflecting the electrokinetic coupling between solvent and ionic flows, such as the electro-osmotic permeability $K_{s}^{\psi}$. Alternatively, for $a+1 /-1$ electrolyte, ionic fluxes can simply be expressed in terms of the salt flux $\bar{J}_{\text {salt }}=\bar{J}_{+}+\bar{J}_{-}$and the electric current $\bar{I}_{\mathrm{el}}=e\left(\bar{J}_{+}-\bar{J}_{-}\right)$, where $e$ is the elementary charge. In that case the coupling matrix takes a symmetric form, reflecting Onsager's symmetry relations $[13,14]$.

\section{B. Pore scale}

On the microscopic scale, the coupling between different transport phenomena arises due to the excess electric charge of the fluid which compensates that of the solid walls. This charge may result in both the acceleration of the whole fluid under an applied electric field (electro-osmosis) and the transport of charge if the fluid flows under an applied pressure gradient (streaming potential). Mass conservation for solvent and ions reads

$$
\partial_{t} \rho_{k}+\nabla \cdot \mathbf{j}_{k}=0
$$

where the flux $\mathbf{j}_{k}$ of species $k \in(s, \pm)$ with respect to the barycentric velocity $\mathbf{u}$ of a fluid element can be expressed as the sum of advection $\rho_{k} \mathbf{u}$ and diffusion-migration $-D_{k} \rho_{k} \nabla \beta \mu_{k}$, with $D_{k}$ the diffusion coefficient of species $k, \mu_{k}$ its local chemical potential, and $\beta=1 / k_{B} T$ the inverse temperature. The chemical potential can further be decomposed into an ideal and an excess part as $\beta \mu_{k}=\ln \rho_{k}+\beta \mu_{k}^{\mathrm{ex}}$, where $\mu_{ \pm}^{\mathrm{ex}}= \pm e \psi$ for the ions. The local electric potential $\psi$ includes both the linearly varying macroscopic potential $\bar{\psi}$ and the local variations due to the charge distribution inside the pore. The resulting conservation law for the ions is the Nernst-Planck equation,

$$
\partial_{t} \rho_{ \pm}+\nabla \cdot\left[\rho_{ \pm} \boldsymbol{u}-D_{ \pm} \nabla \rho_{ \pm} \mp \beta e D_{ \pm} \rho_{ \pm} \nabla \psi\right]=0 .
$$

Momentum conservation implies that the fluid velocity $\boldsymbol{u}$ satisfies the Navier-Stokes (NS) equation,

$$
\partial_{t}\left(\rho^{m} \boldsymbol{u}\right)+\rho^{m}(\boldsymbol{u} \cdot \nabla) \boldsymbol{u}=\eta \Delta \boldsymbol{u}-\nabla P+\boldsymbol{f}^{\mathrm{ext}},
$$

with $\rho^{m}$ and $\eta$ the mass density and dynamic viscosity of the fluid, assumed to be equal to that of the pure solvent, and $P$ the local pressure. The external force $f^{\text {ext }}$ acting on the fluid corresponds to the local force under the effect of the macroscopic gradients $-\nabla \bar{P},-\rho_{\mathrm{el}} \nabla \bar{\psi}$ and $-\left(\rho_{+}+\right.$ $\left.\rho_{-}\right) k_{B} T \nabla \ln \bar{\rho}$. The internal forces arising from the fluid itself can be evaluated from the Gibbs-Duhem equation as

$$
-\nabla P=-\sum_{k} \rho_{k} \nabla \mu_{k}=-\nabla P^{i d}-\rho_{\mathrm{el}} \nabla \varphi
$$

with $\rho_{\mathrm{el}}=e\left(\rho_{+}-\rho_{-}\right)$the local charge density and $\varphi=\psi-$ $\bar{\psi}$ the local electric potential relative to a macroscopic potential $\bar{\psi}$, the gradient of which is the macroscopic electric field in Eq. (1). Both of these quantities further satisfy the Poisson equation,

$$
\nabla^{2} \varphi=-\frac{\rho_{\mathrm{el}}}{\epsilon}
$$


with $\epsilon$ the dielectric permittivity of the aqueous phase. For an incompressible fluid the velocity field further satisfies $\nabla$. $\mathbf{u}=0$. The NS equation (4) then must be solved for given hydrodynamic boundary conditions (e.g., $\mathbf{u}=\overrightarrow{0}$ for nonslip).

\section{Ion distribution on the microscopic scale}

At equilibrium, ionic fluxes vanish and the Nernst-Planck equation (3) results in the Boltzmann distribution,

$$
\rho_{ \pm}=\rho_{\text {ref }} \mathrm{e}^{\mp \beta e \phi}=\rho_{\text {ref }} \mathrm{e}^{\mp \beta e\left(\psi-\psi_{\text {ref }}\right)},
$$

where $\rho_{\text {ref }}$ and $\psi_{\text {ref }}$ are a reference concentration and potential, respectively. In electrically neutral regions such that $\rho_{+}=$ $\rho_{-}$, one has $\psi=\psi_{\text {ref }}$. However, $\rho_{\text {ref }}$ and $\psi_{\text {ref }}$ can be defined even if such regions do not exist in the system. There is no a priori reason for chosing $\bar{\psi}$ as a reference potential, so, in general, the two potentials $\phi$ and $\varphi$ appearing in Eqs. (6) and (7) differ. Nevertheless, this choice can be relevant to make the link between the microscopic and macroscopic scales, as discussed below. Combining Eqs. (6) and (7), one obtains the Poisson-Boltzmann (PB) equation,

$$
\nabla^{2}(\beta e \phi)=\kappa^{2} \sinh (\beta e \phi)
$$

where we have introduced the Debye length $\kappa^{-1}$ such that $\kappa^{2}=8 \pi l_{B} \rho_{\text {ref }}$, with the Bjerrum length $l_{B}=\beta e^{2} / 4 \pi \epsilon$, the distance at which the electrostatic energy between two unit charges is equal to the thermal energy $(0.7 \mathrm{~nm}$ in water at room temperature). When $\beta e \phi \ll 1$, one can linearize the right-hand side of Eq. (8) and the resulting linearized PB equation,

$$
\nabla^{2} \phi=\kappa^{2} \phi
$$

can often be solved analytically. The nonlinear or linearized PB equation then must be solved with suitable boundary conditions. We consider here porous materials with a fixed surface charge density $\sigma e$ (with $\sigma$ in $\mathrm{m}^{-2}$ ), leading to an electric field,

$$
-\nabla \phi \cdot \mathbf{n}=\frac{\sigma e}{\epsilon}
$$

at the surface, with $\mathbf{n}$ the normal unit vector. Note that the present problem has already been addressed in a cylindrical channel under various approximations in the case of a fixed surface potential $\phi_{0}$ (Dirichlet boundary condition, see, e.g., Refs. [45-47]), while Eq. (10) corresponds to a Neumann boundary condition. The latter is physically more relevant for surfaces with a charge density fixed by their chemical composition, such as clay minerals, which are prototypical of the porous materials of interest in the contexts mentioned in the Introduction. In addition, osmotic effects have, to our knowledge, not been considered in that case.

\section{Connecting local and macroscopic variables}

Let us conclude this section by analyzing how the local variables, such as the average salt concentration $\rho_{\text {salt }}$ or some reference potential inside the pore, can be related to the macroscopic ones, i.e., the reference reservoir with concentration $\bar{\rho}$ and potential $\bar{\psi}$. As explained by Moyne and Murad [13,14], it is convenient to reformulate the local problem using auxiliary macroscopic variables. One then can use the macroscopic potential as a reference $\left(\psi_{\text {ref }}=\bar{\psi}\right.$, hence, $\varphi=\phi)$ and consider an electrically neutral reservoir with salt concentration $\bar{\rho}$ in equilibrium with the system. The salt concentration $\rho_{\text {salt }}$ inside the pore then can be linked to the one in the reservoir. For a negative surface charge density $(\sigma e<0)$, the average salt concentration is equal to that of anions: $\rho_{\text {salt }}=\left\langle\rho_{-}\right\rangle=\bar{\rho}\left\langle e^{\beta e \phi}\right\rangle=\bar{\rho} e^{\beta e \psi_{D}}$, where $\psi_{D}$ is the so-called Donnan potential between the system and the reservoir. One can then relate the reservoir concentration and Donnan potentials as

$$
\begin{aligned}
\bar{\rho} & =\sqrt{\rho_{\text {salt }}\left(\rho_{\text {salt }}+\frac{|\sigma| S}{V}\right)}, \\
\psi_{D} & =-\frac{1}{2 \beta e} \ln \left(1+\frac{|\sigma| S}{V \rho_{\text {salt }}}\right)
\end{aligned}
$$

with $V$ the pore volume and $S$ its surface area.

\section{CASE OF A CYLINDRICAL CHANNEL}

\section{A. Ion distributions}

We now restrict ourselves to a cylindrical channel of radius $R$ and assume without loss of generality a negative surface charge density $(\sigma<0)$. The reference concentration $\rho_{\text {ref }}$ and potential $\psi_{\text {ref }}$ then conveniently can be chosen as the values corresponding to the center of the pore $(r=0)$.

In the absence of added salt, i.e., when only counterions are present in the channel, the nonlinear PB equation $\nabla^{2} \phi=$ $-\frac{\rho_{\mathrm{ref} e} e}{\epsilon} e^{-\beta e \phi}$ can be solved analytically. One finds for the potential

$$
\phi(r)=\frac{2}{\beta e} \ln \left(1-\alpha^{2} r^{2}\right)
$$

and for the counterion density profile

$$
\rho_{+}(r)=\frac{2 \alpha^{2}}{\pi l_{B}} \frac{1}{\left(1-\alpha^{2} r^{2}\right)^{2}}
$$

where $\alpha^{-1}$ is the analog of the Debye screening length $\kappa^{-1}$, which can be shown from the electroneutrality condition to satisfy

$$
\alpha^{2} R^{2}=\frac{\pi R|\sigma| l_{B}}{1+\pi R|\sigma| l_{B}} .
$$

In the presence of added salt, only the linearized PB equation (9) can be solved analytically, with the resulting potential

$$
\varphi(r)=\frac{4 \pi l_{B} \sigma}{\kappa \beta e} \frac{I_{0}(\kappa r)}{I_{1}(\kappa R)}
$$

and the ionic density profiles

$$
\rho_{ \pm}(r)=\frac{\kappa^{2}}{8 \pi l_{B}}\left[1 \mp \frac{4 \pi \sigma l_{B}}{\kappa} \frac{I_{0}(\kappa r)}{I_{1}(\kappa R)}\right],
$$

where $I_{n}(x)$ are modified Bessel functions of the first kind. In that case, one finds that the screening length simply satisfies

$$
\kappa^{2}=4 \pi l_{B}\left\langle\rho_{+}+\rho_{-}\right\rangle=4 \pi l_{B}\left(2 \rho_{\text {salt }}+\frac{2|\sigma|}{R}\right),
$$

with $\left\langle\rho_{ \pm}\right\rangle$the average ionic concentrations and $\rho_{\text {salt }}=\left\langle\rho_{-}\right\rangle$ the salt concentration inside the pore. 


\section{B. Flow profiles}

In order to compute the coupling matrix defined by Eq. (1) we need to evaluate the overall solvent and ionic fluxes induced by external pressure, potential, and concentration gradients,

$$
J_{k}^{\gamma}=\frac{1}{\pi R^{2}} \int_{0}^{R} j_{k}^{\gamma} 2 \pi r d r,
$$

where the index $k \in(s, \pm)$ indicates the species and the superscript $\gamma \in(P, \psi, \rho)$ refers to the applied forcing. The solvent flow profile corresponds to the parabolic Poiseuille flow under a pressure gradient, whereas, under an applied electric field, the electro-osmotic flow profile along the channel can be expressed as

$$
v_{z}^{\mathrm{eo}}(r)=-\partial_{z} \bar{\psi} \frac{e}{2 \pi \eta l_{B}} \ln \left(\frac{1-\alpha^{2} r^{2}}{1-\alpha^{2} R^{2}}\right)
$$

in the absence of salt and, under the linearization assumption, as

$$
v_{z}^{\mathrm{eo}}(r)=-\partial_{z} \bar{\psi} \frac{e \sigma}{\eta \kappa}\left[\frac{I_{0}(\kappa r)-I_{0}(\kappa R)}{I_{1}(\kappa R)}\right]
$$

in the presence of salt. In the following, the superscript "eo" will refer to transport of solvent and ions due to the electroosmotic flow. For ions, we will further distinguish the direct effect of the electric field by the superscript "NE" for NernstEinstein.

Finally, a macroscopic concentration gradient, which can be understood as an osmotic pressure gradient, drives the fluid according to a local force $-k_{B} T\left(\rho_{+}+\rho_{-}\right) \nabla \ln \bar{\rho}$. In the linearized PB case, it follows from Eq. (17) that this force is uniform, resulting, as in the case of a pressure gradient, in a parabolic flow, which contributes to the chemico-osmotic (co) transport of both solvent and ions. In order to distinguish the direct effect of the concentration gradient on ion transport (Fick's law) we will use the superscript "d".

\section{Transport coefficients}

Using the results of the previous sections, all terms of the coupling matrix [Eq. (1)] can now be derived for the cylindrical channel. For a pressure gradient, one finds for the solvent the usual permeability

$$
K_{s}^{P}=\frac{\rho^{m} R^{2}}{8 \eta}
$$

and for the ions, in the presence of added salt and under the linearization assumption,

$$
K_{ \pm}^{P}=\frac{\kappa^{2} R^{2}}{64 \pi \eta l_{B}} \mp \frac{\sigma}{2 \eta \kappa} \frac{I_{2}(\kappa R)}{I_{1}(\kappa R)},
$$

while in the absence of salt one obtains (without linearizing)

$$
K_{+}^{P}=\frac{1}{2 \pi \eta l_{B}}\left[1+\frac{\ln \left(1-\alpha^{2} R^{2}\right)}{\alpha^{2} R^{2}}\right] .
$$

In the presence of an applied electric field, the electroosmotic solvent flow corresponds, with salt and under the linearization assumption, to

$$
K_{s}^{\psi}=K_{s}^{\mathrm{eo}}=-\frac{\rho^{m} e \sigma}{\eta \kappa} \frac{I_{2}(\kappa R)}{I_{1}(\kappa R)},
$$

whereas in the absence of salt and without linearization one obtains

$$
K_{s}^{\psi}=K_{s}^{\mathrm{eo}}=\frac{e \rho^{m}}{2 \pi \eta l_{B}}\left[1+\frac{\ln \left(1-\alpha^{2} R^{2}\right)}{\alpha^{2} R^{2}}\right] .
$$

For the ions, $K_{ \pm}^{\psi}=K_{ \pm}^{\mathrm{NE}}+K_{ \pm}^{\text {eo }}$ with the Nernst-Einstein contribution

$$
K_{ \pm}^{\mathrm{NE}}=\left\langle\rho_{ \pm}\right\rangle D_{ \pm} \beta e .
$$

The electro-osmotic term is, in the presence of salt and under the linearization assumption,

$$
K_{ \pm}^{\mathrm{eo}}= \pm \frac{e \sigma^{2}}{2 \eta}-\left[\frac{e \sigma \kappa}{8 \pi \eta l_{B}} \pm \frac{e \sigma^{2}}{2 \eta} \frac{I_{0}(\kappa R)}{I_{1}(\kappa R)}\right] \frac{I_{2}(\kappa R)}{I_{1}(\kappa R)},
$$

while in the absence of added salt and without linearization it reads

$$
K_{+}^{\mathrm{eo}}=\frac{e}{\pi^{2} R^{2} \eta l_{B}^{2}}\left[\frac{\alpha^{2} R^{2}}{1-\alpha^{2} R^{2}}+\ln \left(1-\alpha^{2} R^{2}\right)\right] .
$$

Finally, the concentration gradient induces a solvent flux according to

$$
K_{s}^{\rho}=K_{s}^{\mathrm{co}}=\frac{\rho^{m} \kappa^{2} R^{2}}{32 \pi \eta l_{B}}=\frac{\kappa^{2}}{4 \pi l_{B}} K_{s}^{P}
$$

and ionic fluxes $K_{ \pm}^{\rho}=K_{ \pm}^{d}+K_{ \pm}^{\text {co }}$ with

$$
K_{ \pm}^{d}=\left\langle\rho_{ \pm}\right\rangle \beta D_{ \pm}
$$

and

$$
K_{ \pm}^{\mathrm{co}}=\frac{\kappa^{2}}{4 \pi l_{B}}\left[\frac{\kappa^{2} R^{2}}{64 \pi \eta l_{B}} \mp \frac{\sigma}{2 \eta \kappa} \frac{I_{2}(\kappa R)}{I_{1}(\kappa R)}\right]=\frac{\kappa^{2}}{4 \pi l_{B}} K_{ \pm}^{P} .
$$

\section{LATTICE-BOLTZMANN ELECTROKINETICS}

In this section, we describe the numerical algorithm used to simulate the coupled solvent and ionic transport on the microscopic scale. It is used in what follows to determine the transfer coefficients defining the coupling matrix (1) without resorting to linearization assumptions. The LBE approach combines the lattice-Boltzmann method to enforce momentum conservation in the fluid with a time-dependent density functional theory framework for the evolution of the local composition and the corresponding local forces acting on the fluid $[37,38,40]$. It is based on the kinetic theory whose central quantity is the probability density function $f(\boldsymbol{r}, \boldsymbol{v}, t)$ to find a given particle with a velocity $\boldsymbol{v}$ at a position $\boldsymbol{r}$ and time $t$. We first recall the basic ingredients of the Lattice-Boltzmann method [30]. We then introduce the algorithms used to propagate the ions and to compute the local forces, which are both formulated using the gradient of solute chemical potentials via the NernstPlanck and Gibbs-Duhem equations (see Sec. II B).

\section{A. Momentum conservation}

Numerically, space, time, and velocity space are discretized simultaneously. The fluid particles evolve on a regular lattice of spacing $\Delta x$ composed of nodes $\boldsymbol{r}$ and only a finite set of velocities $\left\{\boldsymbol{c}_{\boldsymbol{i}}\right\}$ are allowed. The hydrodynamic variables are 
computed from the discretized probability density function, or population, $f_{i}(\boldsymbol{r}, t) \equiv f\left(\boldsymbol{r}, \boldsymbol{c}_{\boldsymbol{i}}, t\right)$ as

$$
\rho(\boldsymbol{r}, t)=\sum_{i} w_{i} f_{i}(\boldsymbol{r}, t), \quad \boldsymbol{j}(\boldsymbol{r}, t)=\sum_{i} w_{i} f_{i}(\boldsymbol{r}, t) \boldsymbol{c}_{i},
$$

with $w_{i}$ weights associated to each discrete velocity and ensuring among other the isotropy of the lattice. In the following, we use the D3Q19 lattice [30]. The populations evolve in time steps $\Delta t$ according to a discretized kinetic equation,

$$
\begin{aligned}
& f_{i}\left(\boldsymbol{r}+\boldsymbol{c}_{i} \Delta t, t+\Delta t\right) \\
& \quad=f_{i}(\boldsymbol{r}, t)-\frac{f_{i}(\boldsymbol{r}, t)-f_{i}^{\mathrm{eq}}(\boldsymbol{r}, t)}{\tau}+F_{i}(\boldsymbol{r}) .
\end{aligned}
$$

In this equation, the first term corresponds to the phenomenological BGK (Bhatnagar, Gross, Krook) collision operator describing the relaxation toward a local Maxwell-Boltzmann equilibrium, with a characteristic time $\tau$ controlling the viscosity of the fluid. The second term accounts for the forces acting locally on the fluid. It can be shown by means of a Chapman-Enskog expansion that the hydrodynamic variables then satisfy the Navier-Stokes equation (4). The third term (forcing term) satisfies $\sum_{i} w_{i} F_{i} \boldsymbol{c}_{\boldsymbol{i}}=\boldsymbol{f}$, where the volumetric force $f$ includes both the pressure gradient in the Gibbs-Duhem equation (5), which reflects interactions inside the fluid, and the external forces. Note in particular that the present framework allows us to capture the effect of an external osmotic pressure gradient $-k_{B} T\left(\rho_{+}+\rho_{-}\right) \nabla \ln \bar{\rho}$, which was not considered in previous implementations of the LBE method. The expression of $F_{i}$ is given in the next section.

\section{B. Solute transport and force on the fluid}

The solute densities evolve according to the Nernst-Planck equation (3). Numerically, this can be simulated on the same lattice using the link-flux method. This method, introduced by Capuani et al. $[37,38]$ in order to prevent spurious solute fluxes across solid-fluid interfaces, focuses on solute fluxes between lattice nodes rather than the amount of solute at each node. More precisely, the concentrations are updated as

$$
\frac{\rho_{ \pm}(\mathbf{r}, t+\Delta t)-\rho_{ \pm}(\mathbf{r}, t)}{\Delta t} \Delta x^{3}=-A_{0} \sum_{i} j_{ \pm}^{i}(\mathbf{r}),
$$

where $i$ refers to the discrete velocities and $j_{ \pm}^{i}$ to the contribution of link $i$ between $\mathbf{r}$ and $\mathbf{r}+\boldsymbol{c}_{i} \Delta t$ to the outward flux of $\mathbf{j}_{ \pm}$across the surface of the cell around node $\mathbf{r}$ (for more details, see Ref. [37]).

In order to ensure that the ions follow a Boltzmann distribution at equilibrium, the ionic fluxes are written as $\mathbf{j}_{ \pm}=-D_{ \pm} e^{-\beta \mu_{ \pm}^{\text {ex }}} \nabla\left[\rho_{ \pm} e^{\beta \mu_{ \pm}^{\text {ex }}}\right]$, with $\mu_{ \pm}^{\text {ex }}$ the excess chemical potentials defined in Sec. II B and the link-fluxes expressed in the symmetrized form

$$
\begin{aligned}
j_{ \pm}^{i}(\mathbf{r})= & -d_{ \pm} \frac{e^{-\beta \mu_{ \pm}^{\mathrm{ex}}(\mathbf{r})}+e^{-\beta \mu_{ \pm}^{\mathrm{ex}}\left(\mathbf{r}+\mathbf{c}_{\mathbf{i}} \Delta t\right)}}{2} \\
& \times\left[\frac{\rho_{ \pm}\left(\mathbf{r}+\mathbf{c}_{\mathbf{i}} \Delta t\right) e^{\beta \mu_{ \pm}^{\mathrm{ex}}\left(\mathbf{r}+\mathbf{c}_{\mathrm{i}} \Delta t\right)}-\rho_{ \pm}(\mathbf{r}) e^{\beta \mu_{ \pm}^{\mathrm{ex}}(\mathbf{r})}}{\Delta_{i}}\right]
\end{aligned}
$$

with $d_{ \pm}=D_{ \pm} / A_{0}$ and $\Delta_{i}=\left\|\mathbf{c}_{\mathbf{i}} \Delta t\right\|$. Introducing this expression in Eq. (35), it appears that in this link-flux algorithm the enclosing area does not play an explicit role on the evolution of the densities. For links crossing solid-fluid boundaries (i.e., such that $\mathbf{r}+\mathbf{c}_{\mathbf{i}} \Delta t$ is a solid node) we enforce $j_{ \pm}^{i}=0$ so such links do not carry any solute into the solid. The advective fluxes $\rho_{ \pm} \mathbf{u}$ in Eq. (3) are treated in a separate step described in detail in Ref. [37], which consists in transferring particles according to the overlap between the considered cell (around a node) translated by $\mathbf{u} \Delta t$ and each of its neighboring cells.

The contribution to the forcing term $F_{i}$ in Eq. (34) arising from the presence of solutes is conveniently computed as

$$
F_{i}(\mathbf{r})=-k_{B} T\left[\frac{j_{+}^{i}(\mathbf{r})}{d_{+}}+\frac{j_{-}^{i}(\mathbf{r})}{d_{-}}\right] .
$$

Note that, contrary to previous implementations of the LBE method, in which an ideal contribution was substracted $[37,40]$, we account for the driving of the fluid by local concentration gradients (osmosis). The external force due to a pressure gradient is included as a constant, uniform source with the corresponding $F_{i}=\frac{\mathbf{f} \cdot c_{i}}{c_{s}^{2}}$, with $c_{s}$ the speed of sound of the LB fluid $\left[c_{s}^{2}=\frac{1}{3}\left(\frac{\Delta x}{\Delta t}\right)^{2}\right.$ for the D3Q19 lattice]. The external force due to a potential or concentration gradient is included as a linear contribution to the chemical potentials when computing $F_{i}$ using Eqs. (37) and (36), with a jump at the boundary nodes in order to enforce a continuous gradient consistent with the periodic boundary conditions.

The electrostatic potential $\psi$ is determined on the lattice from the charge distribution by solving the Poisson equation (6) using the successive over-relaxation (SOR) method [48], which also requires to compute the Laplacian of $\psi$. We evaluate the latter using the following stencil:

$$
\nabla^{2} \psi(\mathbf{r})=\frac{2}{\Delta t^{2}} \sum_{i} \frac{w_{i}}{c_{s}^{2}}\left[\psi\left(\mathbf{r}+\mathbf{c}_{\mathbf{i}} \Delta t\right)-\psi(\mathbf{r})\right] .
$$

This choice was motivated by numerical accuracy considerations but also by the fact that it is fully consistent with the discretization used for the LB part of the hybrid scheme.

\section{SIMULATION CONDITIONS}

In this section, we discuss the choice of simulation conditions on the accuracy of the LBE scheme. More precisely, we investigate the effect of the charge distribution inside the solid, the lattice spacing, the value of the surface charge density, and channel radius. The system consists of an infinite cylindrical channel of radius $R$, simulated in a cubic box containing fluid and solid nodes and using periodic boundary conditions in all directions. Non-slip hydrodynamic boundary conditions are implemented using the bounce-back rule at the solid-liquid interface. The simulation parameters are chosen to simulate sodium chloride in water at $T=300 \mathrm{~K}$, with diffusion coefficients $D_{+}=1.3110^{-9} \mathrm{~m}^{2} \mathrm{~s}^{-1}$ and $D_{-}=2.010^{-9} \mathrm{~m}^{2}$ $\mathrm{s}^{-1}$, solvent mass density $\rho^{m}=10^{3} \mathrm{~kg} \mathrm{~m}^{-3}$, viscosity $\eta=$ $10^{-3} \mathrm{~Pa} \mathrm{~s}$, and a dielectric constant $\epsilon_{r}=78.5$. Although the LBE method allows us to compute the full dynamics of the system, we need only the stationary state to determine the transport coefficients of Eq. (1). A steady state for the same hydrodynamic regime (Péclet and Schmidt numbers) 
can be achieved in a much shorter time by reducing the solvent density and increasing the diffusion coefficients by the same scaling factor $\left(10^{2}\right.$ in our case). The final results are then rescaled accordingly. Finally, we consider channels with radii between 2 and $20 \mathrm{~nm}$ and surface charge densities in the range $0.01-0.1 e \mathrm{~nm}^{-2}$, with salt concentrations comprised between 0 and $0.2 \mathrm{~mol}^{-1}$. In practice, the ions are first distributed homogeneously inside the pore and the system is equilibrated in the absence of external forcing. Once the equilibrium (Poisson-Boltzmann) distribution is reached, a pressure, potential, or concentration gradient is imposed and the system is evolved until a steady state is reached. The solvent and ionic fluxes are finally measured in that steady state.

\section{A. Charge distribution inside the solid}

In previous LBE implementations, which were mainly applied to spherical objects, the charge inside the solid was distributed over its whole volume. In the present case, we want to simulate a constant surface charge density. Thus, we first analyze the effect of the charge distribution inside the solid on the resulting concentration profiles inside the fluid. Two sets of simulations are done for a given radius $(R=5 \mathrm{~nm})$ and a moderate surface charge density $\left(\sigma=-0.08 e \mathrm{~nm}^{-2}\right)$, in the absence of added salt. The total charge of the solid is homogeneously distributed either over the whole volume or only over the interfacial solid nodes (see Fig. 1). We use here a lattice spacing $\Delta x=0.208 \mathrm{~nm}$. The effect of lattice spacing will be discussed in the next section. Since we use periodic boundary conditions, the results also depend, in principle, on the width $w$ of the solid matrix around the cylindrical channel (see Fig. 1).

The effect of the charge localization and the width of the solid matrix $(w=\Delta x$ and $50 \Delta x)$ on the counterion profile is presented in Fig. 2, which reports the error $\left(\rho_{+}^{\mathrm{sim}}-\right.$ $\left.\rho_{+}^{\text {analy }}\right) / \rho_{+}^{\text {analy }}$ relative to the analytical result of Eq. (14). The effect of the periodic boundary conditions (with a cubic symmetry) is more pronounced when the width $w$ of the solid matrix is small, as expected. In all cases, the largest deviations from the analytical result are observed near the surface. Moreover, the overall error on the orientationally averaged profiles is much smaller than on the local one, because of (a)

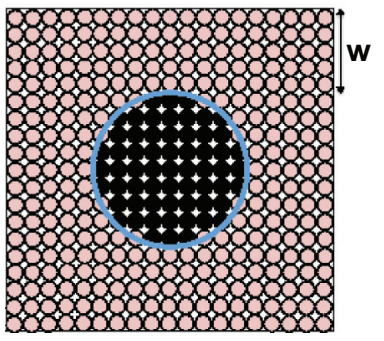

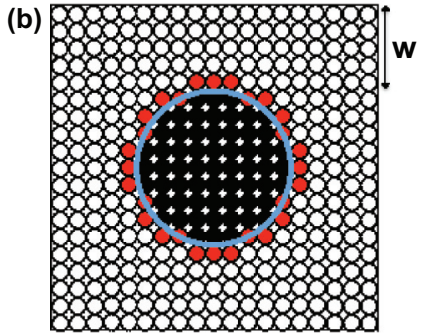

FIG. 1. (Color online) Simulation box for a cylindrical charged pore, using a volumic (a) or surface (b) charge distribution inside the solid. The black circles represent the fluid nodes, and others denote the solid ones. The color of the latter indicate their charge: uncharged (white), weak (pink), or strong (red). Periodic boundary conditions are used in all directions and computed properties may depend on the width $w$ of the solid phase.
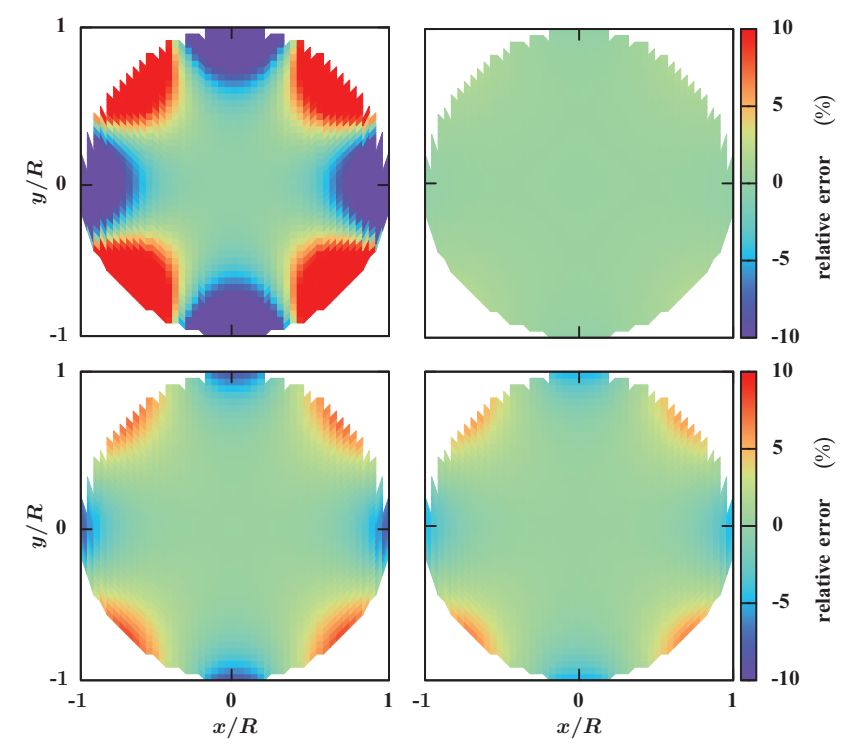

FIG. 2. (Color online) Relative error on the counterion density obtained with the LBE simulations, compared to the analytical result [Eq. (14)] in a cylindrical channel of radius $R=5 \mathrm{~nm}$ and surface charge density $\sigma=-0.08 e \mathrm{~nm}^{-2}$, in the absence of added salt. The simulation results depend on how the charge is distributed inside the solid nodes [in the whole volume (top) or only at the surface (bottom)] and on the size of the solid in which the channel is embedded $[w=\Delta x$ (left) and 50 $\Delta x$ (right), see Fig. 1]. In order to use the same color code for all cases, in the top left part all errors larger than $\pm 10 \%$ appear as red or blue (they can be as large as $\pm 60 \%$ ).

compensations between over- and underestimations. It is worth noticing that for $w=\Delta x$ the error is larger for a volumic charge distribution, while for $w=50 \Delta x$ such a distribution results in the smaller error. Furthermore, the effect of the width $w$ is much more pronounced in the case of a volumic charge distribution.

Importantly, we find that the smallest errors are obtained with a volumic charge distribution in the solid for large $w$. The same conclusions hold for the study of the electro-osmotic flow profile under an applied electric field (not shown). Thus, in the following, we use this configuration for the determination of transport coefficients. Note that as far as the determination of transport coefficient is concerned, the fact that the error is largest close to the surface is partly compensated by the smaller velocity in this region. In fact, the results for all transport coefficients obtained by using a surface charge distribution and a width $w=\Delta x$ (not shown) are nearly identical (the maximum difference observed on all transport coefficients is $0.04 \%$ ) to the one reported below for volumic charge distribution and a width $w=50 \Delta x$ and can be obtained at a much smaller computational cost. Nevertheless, this observation may not be true in arbitrary geometries.

\section{B. Lattice spacing and salt concentration}

The lattice spacing $\Delta x$ is obviously crucial in the control of the accuracy of the LBE scheme for the determination of any property. Here we discuss more quantitatively its influence on the electro-osmotic permeability for cations $K_{+}^{\text {eo }}$, for which the effect is more pronounced than on other quantities 


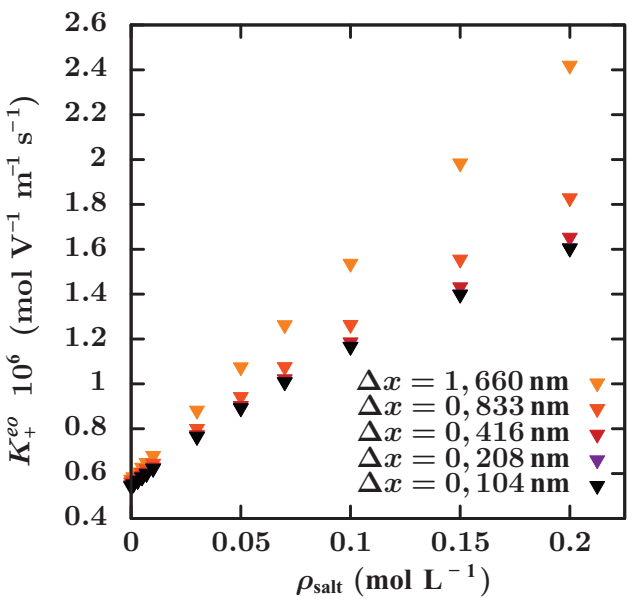

FIG. 3. (Color online) Electro-osmotic permeability for cations $K_{+}^{\mathrm{eo}}$ in a cylindrical channel of radius $R=5 \mathrm{~nm}$ and surface charge density, $\sigma=-0.08 e \mathrm{~nm}^{-2}$, as a function of the salt concentration $\rho_{\text {salt }}$. LBE results are given for different lattice spacings, $\Delta x$.

(e.g., $K_{+}^{\mathrm{NE}}$ or $K_{s}^{\psi}$ ). Figure 3 reports $K_{+}^{\mathrm{eo}}$ as a function of the concentration of added salt $\rho_{\text {salt }}$, for various lattice spacings. Except for $\rho_{\text {salt }}=0$, the analytical results cannot be used as a reference, since they rely on a linearization approximation, the effect of which will be discussed below. The results for $K_{+}^{\text {eo }}$ converge by decreasing the lattice spacing and no difference can be seen on Fig. 3 between the two smallest $\Delta x$. Larger errors due to discretization are observed for larger salt concentrations, for which the screening of the electrostatic potential is stronger and, hence, the ionic and velocity profiles sharper. The criterion for an accurate discretization corresponds to a lattice spacing smaller than the Debye length: Here $\Delta x=0.208 \mathrm{~nm}$ is sufficient and will be used in all the following simulations.

\section{Surface charge and channel radius}

For a given lattice spacing, the accuracy of the numerical simulations also depends on the surface charge and on the channel radius. In the absence of added salt, one can compare the simulated results with the analytical solution of the nonlinear problem. Results for the electro-osmotic permeability for cations $K_{+}^{\text {eo }}$ as a function of the surface charge density $\sigma$ in a cylindrical channel of radius $R=5 \mathrm{~nm}$ are reported in Fig. 4. As expected, $K_{+}^{\text {eo }}$ increases with the surface charge density, as the number of counterions (which accelerate the fluid under an applied field) also increases.

More importantly, the results of LBE simulations are in excellent agreement with the analytical results for the considered conditions, even for large surface charge densities where the linear approximation breaks down. A more systematic study of the error relative to the nonlinear solution, as a function of the surface charge density $\sigma$ and the cylinder radius $R$, in the absence of added salt $\left(\rho_{\text {salt }}=0\right)$, can be found on Figs. 5 and 6 . The former reports the error due to linearization, which increases with both $\sigma$ and $R$ and can be dramatic even for moderate concentrations. The latter reports the error due to discretization within the LBE scheme, which decreases with increasing $R$ and slightly increases with increasing $\sigma$. These

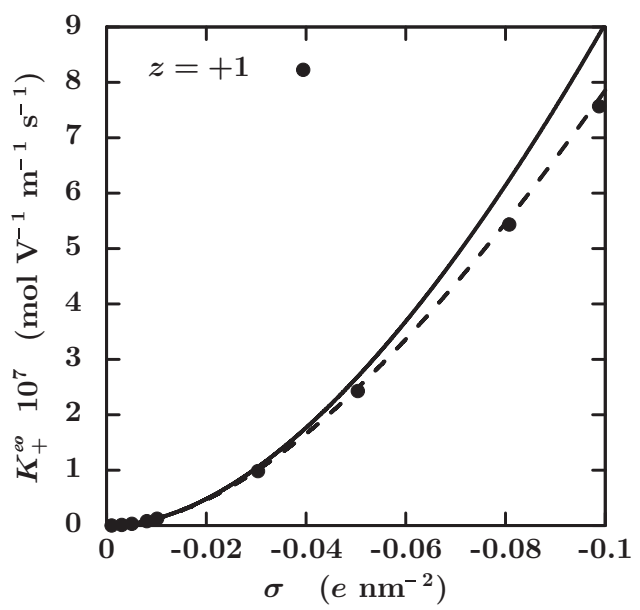

FIG. 4. Electro-osmotic permeability for cations $K_{+}^{\text {eo }}$ as a function of the surface charge density $\sigma$ in a cylindrical channel of radius $R=5 \mathrm{~nm}$, in the absence of added salt $\left(\rho_{\text {salt }}=0\right)$. LBE simulations (symbols) are compared to the analytical results (29) obtained with the nonlinear PB equation (dashed line) and with the linearized one (28) (solid line).

results demonstrate that the linearization approximation is not applicable in most of the considered range, while the LBE simulations are sufficiently accurate to reproduce the nonlinear solution.

\section{TRANSPORT COEFFICIENTS}

From the previous section, we can conclude that the LBE simulations, under the considered conditions and with the choice of simulation parameters described above, allow us to

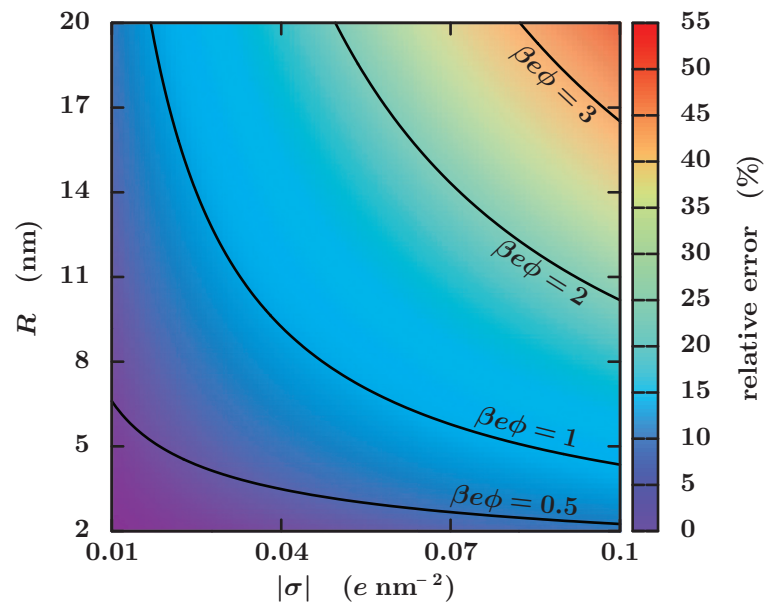

FIG. 5. (Color online) Relative error due to the linearization of the Poisson-Boltzmann equation on the electro-osmotic permeability for cations $K_{+}^{\text {eo }}$, as a function of the surface charge density $\sigma$ and the cylinder radius $R$, in the absence of added salt $\left(\rho_{\text {salt }}=0\right)$. Note that the analytical solution is known for both the linearized [Eq. (28)] and the nonlinear [Eq. (29)] cases. The lines indicate the regions corresponding to given values of the reduced potential $\beta e \phi$, which is assumed to be small in the linearization procedure. As expected, large errors on the transport coefficient $K_{+}^{\text {eo }}$ are obtained when the $\beta e \phi \gtrsim 1$. 


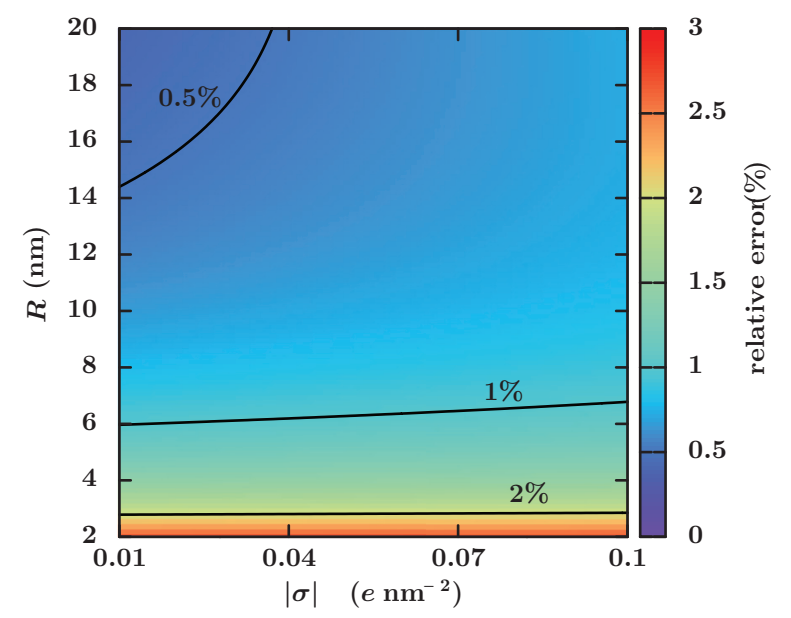

FIG. 6. (Color online) Relative error obtained with the LBE simulations compared to the nonlinear solution [Eq. (29)] for the electro-osmotic permeability for cations $K_{+}^{\text {eo }}$, as a function of the surface charge density $\sigma$ and the cylinder radius $R$, in the absence of added salt $\left(\rho_{\text {salt }}=0\right)$. The simulations are performed with the parameters indicated in the text, in particular a lattice spacing $\Delta x=0.208 \mathrm{~nm}$.

accurately determine numerically the solution of the nonlinear coupled electrokinetic equations and, hence, all the transport coefficients of the coupling matrix (1). We now discuss the latter in more detail for a fixed radius $R=5 \mathrm{~nm}$, emphasizing the role of surface charge and salt concentration. In addition, we analyze the relevance of the linearization approximation for all these transport coefficients.

\section{A. Effect of a pressure gradient}

The solvent flow profile resulting from a pressure gradient is the well-known parabolic Poiseuille flow and does not depend on the surface charge and on the presence of ions. It is well reproduced by the present $\mathrm{LBE}$ approach. Thus, the hydraulic permeability $K_{s}^{P}$ predicted by Eq. (22) is well reproduced (not shown). More interesting is the ion transport induced by this fluid flow. The hydrodynamic permeability $K_{ \pm}^{P}$ for cations and anions is reported in Fig. 7. It increases almost linearly with salt concentration, as more ions are advected by the flow. Note that $K_{+}^{P}$ does not start from zero (contrary to $K_{-}^{P}$ ) as in the absence of added salt the counterion concentration is already $2 \sigma / R \sim 510^{-2} \mathrm{~mol} \mathrm{l}^{-1}$. The linearized results [Eq. (23)] are a rather good approximation of $K_{ \pm}^{P}$ under these conditions. As the velocity profile does not depend of charges in that case, all observed differences originate from the approximation of the ionic profiles only.

\section{B. Effect of a potential gradient}

As mentioned above, the effect of a potential gradient can be decomposed into a trivial direct contribution $K_{ \pm}^{\mathrm{NE}}$ [see Eq. (27)] and the more involved electro-osmotic one $K_{ \pm, s}^{\text {eo }}$ which depends both on the velocity profile induced by the local charge distribution and on the localization of the different species according to their charge. For the solvent flow, there is no direct effect of the electric field: Only the electro-osmotic

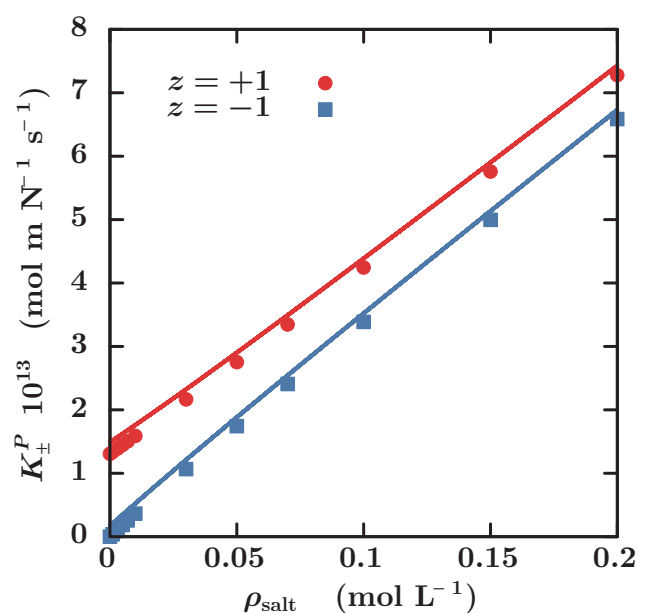

FIG. 7. (Color online) Hydrodynamic permeability $K_{ \pm}^{P}$ (red upper and blue lower lines for cations and anions, respectively), for ions in a cylindrical channel of radius $R=5 \mathrm{~nm}$ and surface charge density $\sigma=-0.08 e \mathrm{~nm}^{-2}$, as a function of salt concentration $\rho_{\text {salt }}$. LBE simulations for cations $(\bullet)$ and anions $(\boldsymbol{\square})$ are compared to the analytical results [Eq. (23)] obtained with the linearized PB equation (line).

flow contributes to $K_{s}^{\psi}$, which is reported as a function of the salt concentration in Fig. 8. The solvent electro-osmotic permeability decreases with increasing salt concentration as a result of the screening of the potential drop across the interface, which results in a smaller fluid velocity. The prediction of the linearized PB theory [Eq. (25)] is rather accurate in that case, as the solvent density profile is homogeneous: Only the effect on the velocity profile matters and this effect is larger in the vicinity of the surface where the contribution to the overall flow is small.

Figure 9 reports the effect of a potential gradient on ionic fluxes. Under these conditions, the major contribution to $K_{ \pm}^{\psi}$ is the direct Nernst-Einstein $K_{ \pm}^{\mathrm{NE}}$ which is well described by Eq. (27) (it does not depend on the density or velocity profiles, only on the average densities). Nevertheless, the

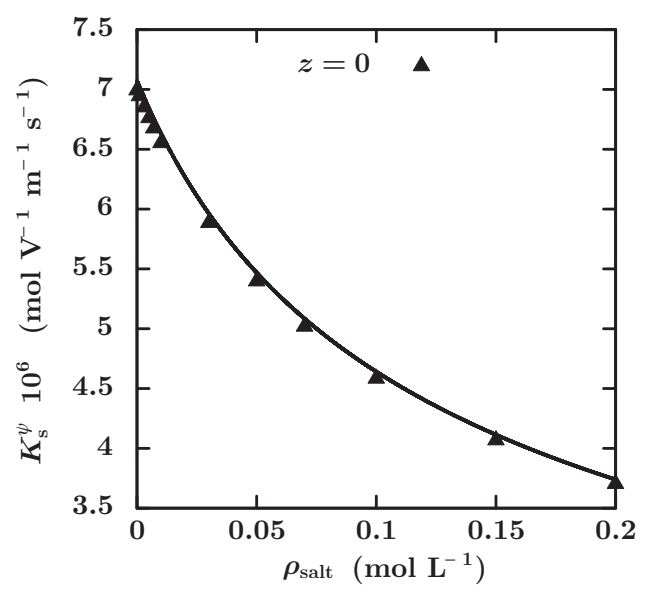

FIG. 8. Electro-osmotic permeability for the solvent $K_{s}^{\psi}$ as a function of salt concentration $\rho_{\text {salt }}$ in a cylindrical channel of radius $R=5 \mathrm{~nm}$. LBE simulations (symbols) are compared to the analytical results [Eq. (25)] obtained with the linearized PB equation (line). 

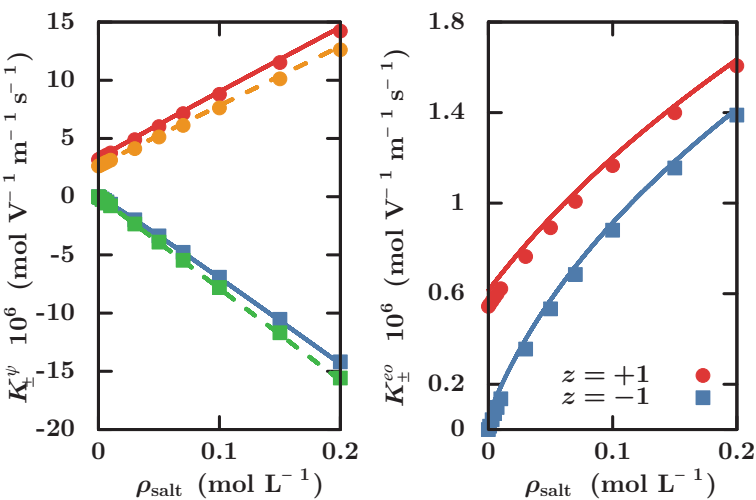

FIG. 9. (Color online) Effect of a potential gradient on the cations ( ) and anions ( $\boldsymbol{\square})$ in a cylindrical channel of radius $R=5 \mathrm{~nm}$ and surface charge density $\sigma=-0.08 e \mathrm{~nm}^{-2}$, as a function of salt concentration $\rho_{\text {salt }}$. The total electrical permeability $K_{ \pm}^{\psi}=K_{ \pm}^{\text {eo }}+K_{ \pm}^{\mathrm{NE}}[($ left $)$ red upper and blue lower solid lines for cations and anions, respectively] as well as the Nernst-Einstein $K_{ \pm}^{\mathrm{NE}}$ [(left) orange upper and green lower dashed lines for cations and anions, respectively] and the electro-osmotic contribution $K_{ \pm}^{\text {eo }}$ [(right) red upper and blue lower solid lines for cations and anions, respectively] are indicated. LBE simulations (symbols) are compared to the analytical results of Eqs. (27) and (28) obtained with the linearized PB equation (lines).

electro-osmotic contribution $K_{ \pm}^{\text {eo }}$ cannot be neglected. It drives both types of ions in the same direction, thus enhancing the cation flux and reducing that of anions (in the opposite direction). Contrary to the solvent case, $K_{ \pm}^{\text {eo }}$ increases with salt concentration, because the corresponding decrease in fluid velocity is not sufficient to balance the larger number of transported ions. The linearized result of Eq. (28) performs better at larger concentrations, because the screening of electrostatic interactions results in smaller potential drops across the interface.

We further examine the effect of linearization by systematically computing the relative error on $K_{+}^{\text {eo }}$ induced by this approximation as a function of the surface charge density and the salt concentration. As can be seen on Fig. 10, this error increases with increasing charge density and decreases with increasing salt concentration, as expected. In the range of explored parameters, the linearization approximation performs rather well but becomes less reliable when the surface charge density is close to $0.1 e \mathrm{~nm}^{-2}$ and the salt concentration is in the $1-10 \mathrm{mmol}^{-1}$ range, which are relevant for natural porous media. In such cases, resorting to the nonlinear result, as determined numerically by the present LBE approach, is recommended. Finally, although the linearization error is small at high salt concentrations, one should also keep in mind that even the nonlinear Poisson-Nernst-Planck is a simplified description, as for such concentrations the nonideality of the system (including, among others, the effect of ionic size), as well as the variation of viscosity or permittivity with salt concentration, must be taken into account.

\section{Effect of a salt concentration gradient}

We finally consider the effect of a concentration gradient. Results for the transport coefficients $K_{s}^{\rho}$ and $K_{ \pm}^{\rho}$ are reported

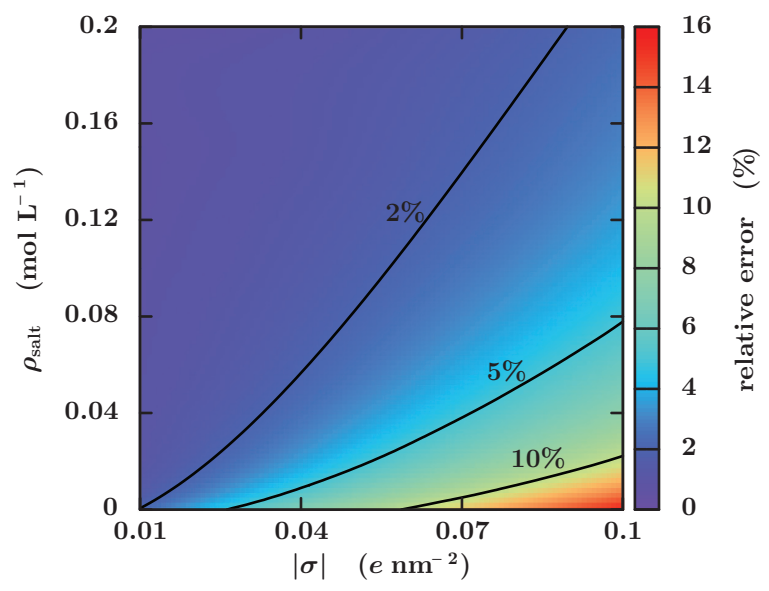

FIG. 10. (Color online) Relative error on the electro-osmotic permeability for cations $K_{+}^{\text {eo }}$ due to the linearization of the PB equation, compared to the nonlinear one (as determined by LBE simulations), as a function of the surface charge density $\sigma$ and the salt concentration $\rho_{\text {salt }}$. The radius of the cylindrical channel is $R=5 \mathrm{~nm}$.

in Fig. 11. They are well described, for the considered surface charge density and channel radius, by the linearized results of Eqs. (30) and (31). In particular, the solvent flux due to a concentration gradient (i.e., an osmotic pressure gradient) is proportional to the ionic concentration inside the channel (which contains counterions even in the absence of added salt). The ionic fluxes contain the trivial Fickian contribution [Eq. (31)], which is proportional to the average ionic concentration and therefore does not rely on the linearization approximation, and the chemo-osmotic one [Eq. (32)], which is more involved but well reproduced by the linear approximation. For small surface charge densities, $K_{ \pm}^{\text {co }}$ grows

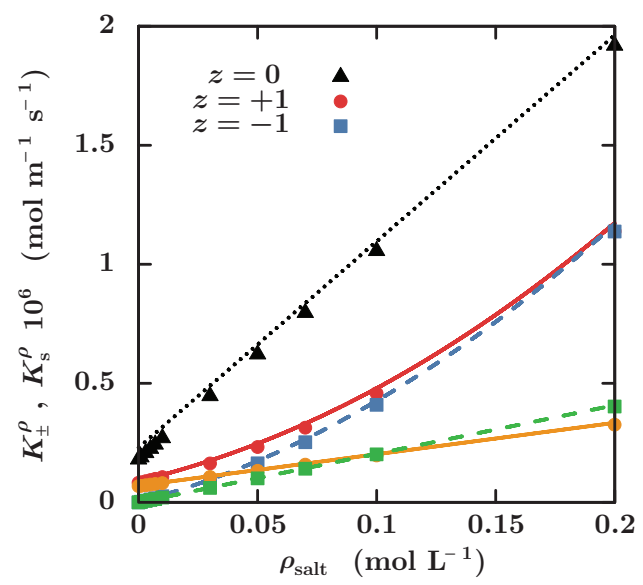

FIG. 11. (Color online) Effect of a concentration gradient on the solvent $(\boldsymbol{\Lambda})$ and ions $(\boldsymbol{\bullet}, \mathbf{\square})$ in a cylindrical channel of radius $R=5 \mathrm{~nm}$ and surface charge density $\sigma=-0.08 e \mathrm{~nm}^{-2}$, as a function of salt concentration $\rho_{\text {salt }}$. For ions, the total coupling parameter $K_{ \pm}^{\rho}=K_{ \pm}^{d}+K_{ \pm}^{\text {co }}$ (red upper solid and blue upper dashed lines for cations and anions, respectively) and the Fickian contribution $K_{ \pm}^{d}$ (orange lower solid and green lower dashed lines for cations and anions, respectively) are indicated. LBE simulations (symbols) are compared to the analytical results [Eqs. (30), (31), and (32)] obtained with the linearized PB equation (lines). 
almost quadratically with the ionic concentration. For small salt concentrations, $K_{ \pm}^{\rho}$ is dominated by the Fickian term $K_{ \pm}^{d}$, while $K_{ \pm}^{\text {co }}$ takes over at large salt concentrations. Comparing Eqs. (31) and (32) one can observe that the crossover between these two regimes depends not only on the surface charge density and the channel radius but also on $R / \beta D_{ \pm} \eta$, which can be interpreted as the ratio between the channel radius and that of the ions (according to the Stokes-Einstein relation $\left.D_{ \pm}=k_{B} T / 6 \pi \eta r_{ \pm}\right)$.

It is worth emphasizing again that previous implementations of the LBE did not account for such osmotic fluxes. Thus, the good agreement between numerical and analytical results, in this regime where linearization is a reasonable approximation (as demonstrated by the analysis of the other transport coefficients), conversely can be seen as a validation of this new feature of the LBE approach. Note that in regimes where the linearization approximation breaks down, the local ionic concentration $\rho_{+}+\rho_{-}$is not homogeneous, so the local force acting on the fluid due to a concentration gradient differs from the homogeneous force arising under the effect of a pressure gradient. In such cases, the velocity profiles is not parabolic and $K_{s, \pm}^{\rho}$ is not be simply related to $K_{s, \pm}^{P}$ as in Eqs. (30) and (32). The LBE simulations nevertheless provide the correct solution.

\section{CONCLUSION}

Mesoscopic lattice-based simulations, such as latticeBoltzmann combined to the link-flux method, can be used to solve numerically the coupled Poisson-Nernst-Planck and Navier-Stokes equations in charged porous media. In the present work, we have introduced an implementation of the lattice-Boltzmann electrokinetics approach which also captures the effect of a macroscopic concentration gradient on the ionic and solvent fluxes. This allowed us to evaluate all the transport coefficients describing the fluxes of solvent and ions in a model porous medium (cylindrical channel) under the effect of macroscopic pressure, potential and salt concentration gradients. We validated this approach by comparison with exact analytical results in the case of no-added salt (only counterions present in the pore) and discussed in detail the influence of simulation parameters (lattice spacing, etc.). Finally, this allowed us to investigate systematically the influence of salt concentration on all transport coefficients (response to pressure, potential, and concentration gradients) in a channel of fixed radius and surface charge density and to assess the validity of analytical results based on the linearization of the Poisson-Boltzmann equation.

Although we have considered here a simple geometry and the stationary state, the present method can be applied to simulate transport in complex porous media and to investigate the full dynamics. In addition, the results for the cylindrical channel can be used as an input for coarser descriptions of transport on larger scales, e.g., in the framework of pore network models, where the void space of the porous medium is represented by an idealized geometry of pore bodies joined by pore throats and which can be adapted to a given experimental structure to compute the transport properties [42-44]. In cases where the linearization approximation is not sufficient, the numerical results of LBE simulations can be introduced in such models. This approach will allow in particular investigation of the Onsager symmetry relations on the sample scale for an arbitrary network of capillaries, including the effect of a concentration gradient and beyond the limit of small double layers.

Finally, it is worth noting that the Poisson-Nernst-Planck approach is also approximate. For the larger concentrations considered here, nonideality of the solution can become nonnegligible. This could be captured in the present framework by introducing an additional term in the excess chemical potential, such as the one from the mean spherical approximation which describes the effect of the finite size of the ions [49,50] or from the fundamental measure theory [51] or by using a modified collision kernel, as introduced by Marconi and Melchionna for the description of confined mixtures [52] and charge transport in nanochannels $[53,54]$. Such lattice-based simulations might also prove useful in addressing the response to pressure, potential, and concentration gradients beyond the linear regime, where nonequilibrium space charge can develop.

\section{ACKNOWLEDGMENTS}

The authors are grateful to Daan Frenkel, Ignacio Pagonabarraga, and Olivier Bernard for useful discussions. A.O. and M.D. acknowledge financial support from Andra. B.R. and M.J. acknowledge financial support from the Agence Nationale de la Recherche under Grant No. ANR-09SYSC-012.
[1] V. Lobaskin, B. Dunweg, M. Medebach, T. Palberg, and C. Holm, Phys. Rev. Lett. 98, 176105 (2007).

[2] D. J. Harrison, K. Fluri, K. Seiler, Z. Fan, C. S. Effenhauser, and A. Manz, Science 261, 895 (1993).

[3] S. van Dorp, U. F. Keyser, N. H. Dekker, C. Dekker, and S. G. Lemay, Nat. Phys. 5, 347 (2009).

[4] G. M. Skinner, M. van den Hout, O. Broekmans, C. Dekker, and N. H. Dekker, Nano Lett. 9, 2953 (2009).

[5] C. K. Haluska, K. A. Riske, V. Marchi-Artzner, J. M. Lehn, R. Lipowsky, and R. Dimova, Proc. Nat. Acad. Sci. USA 103, 15841 (2006).

[6] R. Dimova, K. A. Riske, S. Aranda, N. Bezlyepkina, R. L. Knorr, and R. Lipowsky, Soft Matter 3, 817 (2007).
[7] D. Lacoste, G. Menon, M. Z. Bazant, and J. F. Joanny, Eur. Phys. J. E 28, 243 (2009).

[8] R. R. Thompson, Geophysics 1, 327 (1936).

[9] H. Mizutani, T. Ishido, T. Yokokura, and S. Ohnishi, Geophys. Res. Lett. 3, 365 (1976).

[10] S. Pride, Phys. Rev. B 50, 15678 (1994).

[11] A. Revil and P. Leroy, J. Geophys. Res. Solid Earth 109, B03208 (2004).

[12] J. R. Looker and S. L. Carnie, Transp. Porous Med. 65, 107 (2006).

[13] C. Moyne and M. A. Murad, Transp. Porous Med. 62, 333 (2006).

[14] C. Moyne and M. A. Murad, Transp. Porous Med. 63, 13 (2006). 
[15] G. Allaire, A. Mikelic, and A. Piatnitski, J. Math. Phys. 51, 123103 (2010).

[16] P. Mazur and J. T. G. Overbeek, Recl. Trav. Chim. 70, 83 (1951).

[17] E. Brunet and A. Ajdari, Phys. Rev. E 69, 016306 (2004).

[18] D. Coelho, M. Shapiro, J. F. Thovert, and P. M. Adler, J. Colloid Interface Sci. 181, 169 (1996).

[19] S. Marino, M. Shapiro, and P. Adler, J. Colloid Interface Sci. 243, 391 (2001).

[20] A. K. Gupta, D. Coelho, and P. M. Adler, J. Colloid Interface Sci. 303, 593 (2006).

[21] A. Gupta, D. Coelho, and P. Adler, J. Colloid Interface Sci. 319, 549 (2008).

[22] I. Pagonabarraga, B. Rotenberg, and D. Frenkel, Phys. Chem. Chem. Phys. 12, 9566 (2010).

[23] J. F. Dufrêche, V. Marry, N. Malikova, and P. Turq, J. Mol. Liq. 118, 145 (2005).

[24] L. Joly, C. Ybert, E. Trizac, and L. Bocquet, J. Chem. Phys. 125 204716 (2006).

[25] D. M. Huang, C. Cottin-Bizonne, C. Ybert, and L. Bocquet, Phys. Rev. Lett. 98, 177801 (2007).

[26] D. M. Huang, C. Cottin-Bizonne, C. Ybert, and L. Bocquet, Langmuir 24, 1442 (2008).

[27] L. Bocquet and E. Charlaix, Chem. Soc. Rev. 39, 1073 (2010).

[28] A. Botan, B. Rotenberg, V. Marry, P. Turq, and B. Noetinger, J. Phys. Chem. C 115, 16109 (2011).

[29] A. Botan, V. Marry, B. Rotenberg, P. Turq, and B. Noetinger, J. Phys. Chem. C 117, 978 (2013).

[30] S. Succi, The Lattice Boltzmann Equation for Fluid Dynamics and Beyond (Oxford University Press, Oxford, 2001).

[31] S. Chen and G. D. Doolen, Annu. Rev. Fluid Mech. 30, 329 (1998).

[32] A. J. C. Ladd and R. Verberg, J. Stat. Phys. 104, 1191 (2001).

[33] H. Chen, S. Kandasamy, S. Orszag, R. Shock, S. Succi, and V. Yakhot, Science 301, 633 (2003).

[34] E. S. Boek and M. Venturoli, Comput. Math. Appl. 59, 2305 (2010).
[35] R. Ledesma-Aguilar, R. Nistal, A. Hernandez-Machado, and I. Pagonabarraga, Nat. Mater. 10, 367 (2011).

[36] J. Zhang, Microfluid. Nanofluid. 10, 1 (2011).

[37] F. Capuani, I. Pagonabarraga, and D. Frenkel, J. Chem. Phys. 121, 973 (2004).

[38] I. Pagonabarraga, F. Capuani, and D. Frenkel, Comput. Phys. Commun. 169, 192 (2005)

[39] B. Rotenberg, I. Pagonabarraga, and D. Frenkel, Europhys. Lett. 83, 34004 (2008).

[40] B. Rotenberg, I. Pagonabarraga, and D. Frenkel, Faraday Discuss. 144, 223 (2009).

[41] G. Giupponi and I. Pagonabarraga, Phys. Rev. Lett. 106, 248304 (2011).

[42] D. Bauer, S. Youssef, M. Han, S. Bekri, E. Rosenberg, M. Fleury, and O. Vizika, Phys. Rev. E 84, 011133 (2011).

[43] D. Bauer, S. Youssef, M. Fleury, S. Bekri, E. Rosenberg, and O. Vizika, Transp. Porous Med. 94, 505 (2012).

[44] C. Varloteaux, M. T. Vu, S. Békri, and P. M. Adler, Phys. Rev. E 87, 023010 (2013).

[45] C. L. Rice and R. Whitehead, J. Phys. Chem. 69, 4017 (1965).

[46] S. Levine, J. Marriott, G. Neale, and N. Epstein, J. Colloid Interface Sci. 52, 136 (1975).

[47] W. Olivares, T. L. Croxton, and D. A. McQuarrie, J. Phys. Chem. 84, 867 (1980).

[48] W. H. Press, S. A. Teukolsky, W. T. Vetterling, and B. P. Flannery, Numerical Recipes in C: The Art of Scientific Computing, 2nd ed. (Cambridge University Press, Cambridge, 1993).

[49] J.-F. Dufrêche, O. Bernard, S. Durand-Vidal, and P. Turq, J. Phys. Chem. B 109, 9873 (2005).

[50] M. Jardat, J.-F. Dufrêche, V. Marry, B. Rotenberg, and P. Turq, Phys. Chem. Chem. Phys. 11, 2023 (2009).

[51] D. Gillespie, W. Nonner, and R. S. Eisenberg, J. Phys.: Condens. Matter 14, 12129 (2002).

[52] U. Marini Bettolo Marconi and S. Melchionna, J. Chem. Phys. 131, 014105 (2009).

[53] S. Melchionna and U. Marini Bettolo Marconi, Europhys. Lett. 95, 44002 (2011).

[54] U. Marini Bettolo Marconi and S. Melchionna, Langmuir 28, 13727 (2012) 